\title{
Performance Evaluation of Distributed Protocols Using Different Levels of Heterogeneity Models in Wireless Sensor Networks
}

\author{
Samayveer Singh, Satish Chand, and Bijendra Kumar \\ Department of Computer Engineering \\ Netaji Subhas Institute of Technology, New Delhi, India \\ samayveersingh@gmail.com, schand86@hotmail.com, and bizender@gmail.com
}

\begin{abstract}
Most of the protocols for enhancing the lifetime of wireless sensor networks (WSNs) are of a homogeneous nature in which all sensors have equal amount of energy level. In this paper, we study the effect of heterogeneity on the homogeneous protocols. The ALBPS and ADEEPS are the two important homogeneous protocols. We incorporate heterogeneity to these protocols, which consists of 2-level, 3-level and multi-level heterogeneity. We simulate and compare the performance of the ALBPS and ADEEPS protocols in homogeneous and heterogeneous environment. The simulation results indicate that heterogeneous protocols prolong the network lifetime as compared to the homogeneous protocols. Furthermore, as the level of heterogeneity increases, the lifetime of the network also increases.
\end{abstract}

Index Terms - Wireless sensor networks, heterogeneity, sensor nodes, sensing range, targets.

\section{INTRODUCTION}

A wireless sensor network (WSN) consists of low-size and low-complex devices, which are called as sensor nodes. A sensor can sense the environment to gather information from the monitoring field and communicate through wireless links. The data collected by a sensor is forwarded via multiple hops to a particular node, called as a sink. The sink can utilize the received data or it can forward to other networks connected through internet. A sensor node can be in one of the states: stationary or moving. It may or may not have information about its location. The network can be homogeneous or heterogeneous. In a homogeneous network, all sensor nodes have the same amount of energy. A nonhomogeneous network is called heterogeneous network. A WSN can be used in several applications such as national security, military, healthcare, environmental monitoring, temperature, humidity, vehicular movement, pressure, soil makeup, noise levels, presence or absence of certain types of objects [3]. Sensor network applications can be divided into two groups: querying applications and tasking applications. In querying applications, the information collected by sensors is processed based on the query that triggers the data collection action; for example, collecting the data about an event in the monitoring environment. In tasking applications, the data collection is triggered by an event that is to be observed by the network. An event triggering forces the sensor node to perform some action. The data aggregation is done in order to avoid duplication of data as the adjacent nodes may send some identical data to sink. Sensors can also be coordinated to get a better idea about the event; for example, some sensors can be moved closer to the event.

One of the most important features of a sensor network is its network lifetime. For enhancing the network lifetime, there have been discussed some protocols that include ALBPS and ADEEPS. These protocols assume the network to be homogenous. In ALBPS, the network is kept alive as long as possible by means of load-balancing. In ADEEPS, a low energy sensor tries to minimize its energy consumption rate for targets while allowing higher energy consumption for sensors with higher energy. The network lifetime can be increased by power saving techniques. These techniques may be classified in two categories: scheduling the sensor nodes to alternate between active and sleep mode, and adjusting the sensing range in wireless sensor nodes. Placing few heterogeneous nodes in the sensor network can bring the following three main benefits [6]: prolonging network lifetime, improving reliability of data transmission and decreasing latency of data transportation.

In this work, we concentrate on maximizing sensor network lifetime by using ALBPS and ADEEPS algorithms incorporated with different level of energy heterogeneity in sensor nodes. We make comparative analysis of homogeneous and heterogeneous versions of ALBPS and ADEEPS protocols. In this work, we use 2level, 3-level and multi-level heterogeneity. The heterogeneous versions of ALBPS and ADEEPS have better performance than their homogeneous versions. Moreover, as the level of heterogeneity increases, the lifetime of the network also increases.

The rest of the paper is organized as follows. Section II discusses the literature survey. The proposed work is discussed in Section III and the simulation results are presented in section IV. Finally the paper is concluded in Section V. 


\section{LITERATURE SURVEY}

In this section, we review the related existing works on improving the lifetime of wireless sensor networks. We consider three aspects in reference to improving the lifetime of WSNs that include scheduling algorithms, adjusting sensing range and heterogeneity of sensors.

In [1], Berman et al. discuss centralized and distributed algorithms for increasing the lifetime of sensor networks. These algorithms solve the area coverage problem and SNLP (Sensor Network Lifetime Problem). They use scheduling approach of sensor nodes and a method for solving the fixed range maximum lifetime problem. In this method, the sensing range cannot be changed and it is meant for homogeneous WSNs. Cardei et al. discuss another approach to extend the sensor network lifetime by organizing the Sensors into a maximal number of set covers that are activated successively. Only the sensors from the current active set are responsible for monitoring all targets. In their work they have provided the solution of the maximum set covers (MSC) problem and designed two heuristics to efficiently compute the cover sets [2]. In [4], Brinza and Zelikovsky discuss two distributed target monitoring protocols, namely Load-Balancing Protocol and DEEPS Protocol for lifetime problem. These protocols solve the target coverage problem by scheduling and fixed sensing range approach. Their work is an extension of distributed algorithm, discuss in [1]. Cardei et al. [3] address the target coverage problem with scheduling model and adjustable sensing range. In that work, adjustable range set covers (AR-SC) problem is solved by finding a maximum number of set covers and the ranges associated with each sensor in such a way that each sensor set covers all targets. A sensor can participate in multiple sensor cover sets. Dhawan et al. improve the lifetime of WSNs using both scheduling and adjusting sensing ranges. They discuss two completely localized and distributed scheduling algorithms such as ALBPS and ADEEPS with adjustable sensing range for coverage problem [5]. These algorithms may be considered as an enhancement of distributed algorithms for fixed sensing range discussed in [4].

Besides the scheduling and the adjustable sensing range in our work, we consider the heterogeneous energy levels such as 2-Level, 3- Level and multi-Level in sensor network [6-15]. We consider two scenarios that are for load balancing protocol and deterministic energy efficient protocol for WSNs. In both the scenarios the lifetime of the sensor network increases significantly using 2-level, 3-level and multilevel heterogeneity. As the level of heterogeneity increases, the lifetime of a WSN also increases. In the next section we discuss our proposed work.

\section{PROPOSED WORK}

Our proposed work is based on the ALBPS and ADEEPS protocols. These protocols consider three states for a sensor i.e, a sensor node can be in one of the three states: active, idle and deciding. In active state a sensor monitors the targets and in the idle / sleep state it conserves the energy. In the deciding state, it monitors targets and can change its current state. In ALBPS, a sensor in the deciding state with range $r$ changes its current state to either active or idle state depending on its energy level for load balancing. It is also possible that it may not change its current state until the target is covered by any other active sensor node. In ADEEPS, there must be at least one sensor in-charge of each target. When a sensor is in the deciding state with range $r$, it changes its current state to either active or idle state. Whenever a sensor is not in-charge of any target, it switches to idle state [4]. An active sensor stays in that state for a certain period of time called shuffle time. In order to find its cover schedule, each sensor initially broadcasts its current energy level and covered targets to all neighbors, and then changes its current state to the deciding state with its maximum sensing range. During the shuffle time the active sensors update their energy levels.

We extend ALBPS and ADEEPS protocols by incorporating heterogeneity of various levels. The heterogeneity levels that are considered include 2-level, 3-level and multi-level heterogeneity. In these levels, the sensor nodes have different amounts of energy. In 2-level heterogeneity there are two types of sensor nodes and in 3-level there are three types of sensor nodes. In multilevel heterogeneity the energy is randomly distributed from a given energy interval to sensor nodes. Details of these levels of heterogeneity are given below.

\section{A. 2-Level Heterogeneity-}

In 2-level heterogeneity, there are two types of sensor nodes: advanced and normal nodes. Consider $\mathrm{N}$ sensor nodes that are deployed in a given rectangular area. Let $\mathrm{E}_{0}$ be the initial energy of a normal node and $\mathrm{m}$ be the fraction of the advanced nodes, which own a times more energy than the normal ones. Thus there are $\mathrm{m} * \mathrm{~N}$ advanced nodes equipped with initial energy of $\mathrm{E}_{0} *$ $(1+\mathrm{a})$, and $(1-\mathrm{m}) * \mathrm{~N}$ normal nodes equipped with initial energy of $E_{0}$. The total energy for 2-level heterogeneity, denoted by $E_{2 \text { total }}$, of the network [8] is given by

$$
\begin{aligned}
\mathrm{E}_{2 \text { total }} & =\mathrm{N} *(1-\mathrm{m}) * \mathrm{E}_{0}+\mathrm{N} * \mathrm{~m} * \mathrm{E}_{0} *(1+\mathrm{a}) \\
& =\mathrm{N} * \mathrm{E}_{0} *(1+\mathrm{am})
\end{aligned}
$$

\section{B. 3-level Heterogeneity-}

In 3-level heterogeneity, there are three types of sensor nodes: super, advanced and normal nodes. Consider $\mathrm{m}$ fraction of $\mathrm{N}$ as advance nodes and $\mathrm{m} 0$ fraction of the advance nodes as super nodes. The normal nodes have $\mathrm{E}_{0}$ as initial energy. The energies of advance and super nodes are respectively and $\beta$ times more than that of the normal nodes. Thus energies of the each super and advanced nodes are $\mathrm{E}_{0} *(1+\beta)$ and $\mathrm{E}_{0} *(1+\mathrm{a})$ respectively. The total energy for 3 -level heterogeneity, 
denoted by $\mathrm{E}_{3 \text { total }}$, of the networks $[6,7]$ is given by

$$
\begin{gathered}
\mathrm{E}_{3 \text { total }}=\mathrm{N} *(1-\mathrm{m}) * \mathrm{E}_{0}+\mathrm{N} * \mathrm{~m} *\left(1-\mathrm{m}_{0}\right) * \mathrm{E}_{0} \\
\quad *(1+\mathrm{a})+\mathrm{N} * \mathrm{~m} * \mathrm{~m}_{0} * \mathrm{E}_{0} *(1+\beta) \\
\mathrm{E}_{3 \text { total }}=\mathrm{N} * \mathrm{E}_{0} *\left(1+\mathrm{m} *\left(\mathrm{a}+\mathrm{m}_{0} * \beta\right)\right)
\end{gathered}
$$

\section{Multi-Level Heterogeneity-}

In multi-level heterogeneity, the initial energy of sensor nodes is randomly allocated from the given energy interval $\left[\mathrm{E}_{0}, \mathrm{E}_{0} *\left(1+\mathrm{a}_{\max }\right)\right]$, where $\mathrm{E}_{0}$ is the lower bound of the energy interval and $a_{\max }$ determines the upper bound of the energy interval. Initially, the node $s_{i}$ is equipped with the initial energy of $E_{0} *\left(1+a_{i}\right)$, which is $a_{i}$ times more energy than the lower bound $E_{0}$ of the energy interval. The total initial energy of the network with multi-level heterogeneity [8], denoted by $E_{\text {mtotal }}$, is given by

$$
\begin{aligned}
\mathrm{E}_{\text {mtotal }} & =\sum_{\mathrm{i}=1}^{\mathrm{N}} \mathrm{E}_{0} *\left(1+\mathrm{a}_{\mathrm{i}}\right) \\
& =\mathrm{E}_{0} *\left(\mathrm{~N}+\sum_{\mathrm{i}=1}^{\mathrm{N}} \mathrm{a}_{\mathrm{i}}\right)
\end{aligned}
$$

We now discuss our proposed work. In 2-level heterogeneity, initially the advance nodes are active nodes and they monitor the targets. If any target is not covered by the advanced nodes then some of the normal nodes that can monitor uncovered targets become active nodes. When during the reshuffle time the energy level of the active nodes becomes less than that of the normal nodes, then some of the normal nodes that can cover all targets become active.

In 3-level heterogeneity, initially the super nodes are active nodes and they cover the targets. In case some target is not covered by the super nodes, then some of the advance nodes that can monitor uncovered targets become active nodes. If some targets are still not covered by super/advance nodes, then some of the normal nodes that can monitor the uncovered targets become active nodes. When during the reshuffle time the energy level of the active nodes becomes less than that of the advance nodes (in case there are not active nodes) or normal nodes (which are not active nodes). These active nodes are replaced by the advance nodes. When the energy level of the active nodes becomes less than that of the super nodes (in active) or advanced nodes or normal nodes (in this order) then some of the super/advanced/normal nodes that can cover all targets become active nodes.

In Multi-level heterogeneity, initially the highest energy level nodes are active that cover all targets. If some targets are not coved by the highest energy level nodes, the nodes having less energy than the highest energy nodes that can cover the remaining targets become active. This process continues in a similar way as discuss for 3-level heterogeneity.

Here, we determine the cover sets by using the active nodes. The number of cover sets depend on the number of targets, sensor and target positions. We try to have minimum number of active sensors for monitoring all targets in a cover set so that minimum energy is used to monitor all targets. Other sensors become idle (go to idle states). Each active sensor broadcasts its covered targets, energy level and information about the change of states. If an active sensor nearly exhausts its energy supply and is going to die $\left(E_{\text {total }}=0\right)$, it informs its neighbors about its energy level. A subset of neighbors which are in idle state changes to active states by replacing the exhausted sensor.

The proposed work consists of two algorithms which are based on load balancing and energy efficiency using set target in-charge. Their steps are given as follows:

Step1: Information about the sensor nodes and targets are stored in two vectors denoting as vectors $\left\langle\mathrm{S}_{\mathrm{i}}\right\rangle$ and $\left\langle\mathrm{T}_{\mathrm{j}}\right\rangle$ for sensor $\mathrm{S}_{\mathrm{i}}$ and target $\mathrm{T}_{\mathrm{j}}$, respectively. The vector $\left\langle\mathrm{S}_{\mathrm{i}}\right\rangle$ contains node $\mathrm{id}$, total energy and $\mathrm{x}$, y position of $\mathrm{i}^{\text {th }}$ sensor. The vector $\left\langle\mathrm{T}_{\mathrm{j}}\right\rangle$ contains target $\mathrm{id}$ and $\mathrm{x}, \mathrm{y}$ coordinates of $\mathrm{j}^{\text {th }}$ target.

Step2: Total energy of the network for 2-level, 3-level and multi-level heterogeneity is computed using (1), (2) and (3), respectively.

Step3: Construct the cover sets using the method discussed in [3] for all the targets by using Euclidean distance and sensing range in such a way each target is covered by at least one sensor.

Step 4: Any node which is in a deciding state takes decision to go to sleep or active states.

Step5: Each sensor knows its neighbouring sensors and covered targets. We try to reduce the sensing range of each sensor without leaving any target uncovered by the sensors (in step 5a) and decide the in-charge sensor (in step 5b).

Step 5a: For each sensor, check the target covered at the highest range. If that target is covered by any of the neighboring sensor with highest energy (more than the active sensor), that target is moved from neighbourhood of the active sensor to that of the neighboring sensor i.e, the target is monitored by the neighboring sensor. This process continues for all targets of the active sensor. If the range reaches to zero i.e the active sensor does not cover any target, it goes to sleep state. This process is called load balancing approach [4].

Step 5b: A sensor among all sensors covering a given target that has maximum energy acts as an in-charge for that target. The sensor in-charge of target should not change its state to sleep state until it discovers that that target is covered by another active sensor. This process stops when all sensors have made decisions. This is called energy efficient approach [4].

Step 6: each sensor stays in its current state for a definite time, called shuffle time or the energy level of the active sensor is exhausted.

Step 7: Update energy of each active sensor according to its sensing range after shuffle time as per energy model employed.

Step8: repeat steps 3-7 till all targets are covered by active sensors. If some target is uncovered, the network fails. 
The load balancing and energy efficient algorithms have been adapted for 2-level, 3-level and multi-level heterogeneity.

\section{SimULATION RESULTS}

Our proposed work consists of ALBPS and ADEEPS with different level of heterogeneity. We have considered 2-level, 3- level and multi-level heterogeneity for both the protocols ALBPS and ADEEPS. Further we have considered two types of energy models: linear and quadratic [4].

The linear model is defined as $\mathrm{e}_{\mathrm{p}}=\mathrm{c}_{1} * \mathrm{r}_{\mathrm{p}}$, where $\mathrm{c} 1$ is constant that is defined as

$$
\mathrm{c}_{1}=\frac{\mathrm{E}}{\left(\sum_{\mathrm{r}=1}^{\mathrm{P}} \mathrm{rp}\right)}
$$

and $e_{p}$ is the energy needed to cover a target at distance $r_{p}$ The quadratic model is defined as $\mathrm{e}_{\mathrm{p}}=\mathrm{c}_{2} * \mathrm{r}_{\mathrm{p}}^{2}$ where $\mathrm{c}_{2}$ is a constant that is given by

$$
\mathrm{c}_{2}=\frac{\mathrm{E}}{\left(\sum_{\mathrm{r}=1}^{\mathrm{P}} \mathrm{r}_{\mathrm{p}}^{2}\right)}
$$

For 2-level heterogeneity, two scenarios have been considered by taking the values of $(\mathrm{m}, \mathrm{a})$ as $(0.2,3) \&$ $(0.1,5)$. In other words, in first scenario, $20 \%$ of nodes have been taken as advanced nodes that are equipped with $300 \%$ more energy than the normal nodes, and in second scenario $10 \%$ of the nodes have been taken as advanced nodes that are equipped with $500 \%$ more energy than normal nodes. For 3-level heterogeneity also, two scenarios have been considered by taking the values of $(\mathrm{a}, \beta, \mathrm{m}, \mathrm{m} 0)$ as $(1.5,3,0.5,0.4) \&(1,3,0.2,0.5)$. In first scenario, $30 \%$ of the nodes have been taken as advanced nodes that are equipped with $150 \%$ and $20 \%$ of the nodes as super nodes that are equipped with $300 \%$ more energy than the normal nodes. In second scenario $10 \%$ of the nodes have been considered as advanced nodes that are equipped with $100 \%$ and $10 \%$ of the nodes as super nodes that are equipped with $300 \%$ more energy than the normal nodes. For multilevel heterogeneity the values of the energy interval $\left[\mathrm{E}_{0}, \mathrm{a}_{\max }\right]$ have been taken as $[0.5,2]$. The input parameters used in our simulations are given in Table I.

Table 1. Simulation Parameters

\begin{tabular}{|c|c|c|}
\hline Parameters & Symbols & Values \\
\hline Number of Sensors & $S$ & $40 \sim 200$ \\
\hline Number of Targets & $T$ & $25 \& 50$ \\
\hline Sensor initial energy & $E_{i}$ & $0.5 \mathrm{~J}$ \\
\hline $\begin{array}{c}\text { Adjustable Sensing } \\
\text { Ranges }\end{array}$ & $P\left(r_{1}, r_{2}\right)$ & $30 m$ and $60 m$ \\
\hline Communication Range & $r$ & $2 *$ sensing range \\
\hline $\begin{array}{c}\text { 2 level heterogeneity } \\
\text { Case I \& Case II }\end{array}$ & $m, a$ & $(0.2,3) \&(0.1,5)$ \\
\hline $\begin{array}{c}\text { 3 level heterogeneity } \\
\text { Case I \& Case II }\end{array}$ & $\begin{array}{c}(a, \beta, m, \\
\left.m_{0}\right)\end{array}$ & $\begin{array}{c}(1.5,3,0.5,0.4) \& \\
(1,3,0.2,0.5)\end{array}$ \\
\hline $\begin{array}{c}\text { Multi- level } \\
\text { heterogeneity }\end{array}$ & {$\left[E_{0}, a_{\max }\right]$} & {$[0.5,2]$} \\
\hline
\end{tabular}

We have implemented our proposed protocols in $\mathrm{C}++$. The results are discussed using three types of graphs by varying the number of sensors, maximum sensing range, and maximum sensing range along with number of targets. First we discuss the effect of changes in number of sensors on the lifetime. Figs. 1(a) \& 2(a) show the lifetime of the sensor network by using linear model and Figs. 1(b) \& 2(b) show for quadratic model with respect to number of sensor nodes varying from 40 to 200 with an increment of 20 for 50 targets using ALBPS protocol with heterogeneity. In Figs. $1 \& 2$, simulation is conducted for $30 \mathrm{M}$ and $60 \mathrm{M}$ sensing range respectively and other parameters have been kept same. It is evident from Figs. $1 \& 2$ that the lifetime increases as the number of sensors increases. It is logically justified because increasing the number of sensors increases the energy of the network and hence the lifetime. Furthermore the ALBPS with heterogeneity performs better than original ALBPS. In fact, as the level of heterogeneity increases, the lifetime also increases.
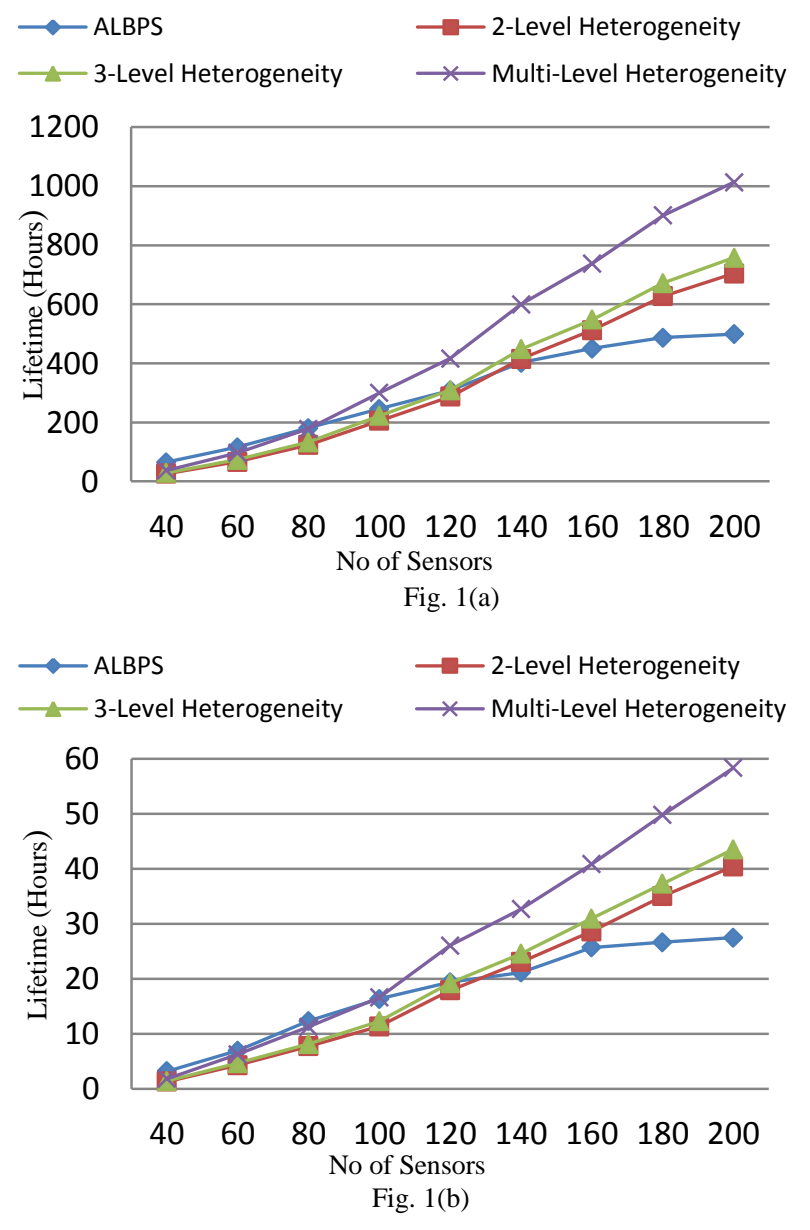

Fig. 1(a) \& (b): The total network Lifetime for different number of sensors at $30 \mathrm{M}$ adjustable sensing range and 50 Targets for Linear and

Quadratic energy model $(\mathrm{m}=0.2$ and $\mathrm{a}=3$ for 2 -level; $\mathrm{a}=1.5, \beta=3$, $\mathrm{m}=0.5$ and $\mathrm{m} 0=0.4$ for 3 -level)

We now discuss the effect of sensing range on the lifetime of sensor networks for the given number of sensors. The sensors are categorized based on the parametric values as given in Table I (case I and case II for 2-level and 3-level). We have deployed 200 number 
of sensors out of which 160 are normal and 40 advanced nodes for 2-level heterogeneity. An advance node has 3 times more energy than a normal node. In case of threelevel heterogeneity, there are 40 super nodes equipped with 3 times more energy than the normal nodes and 60 advanced nodes deployed with 1.5 times more energy than normal nodes.
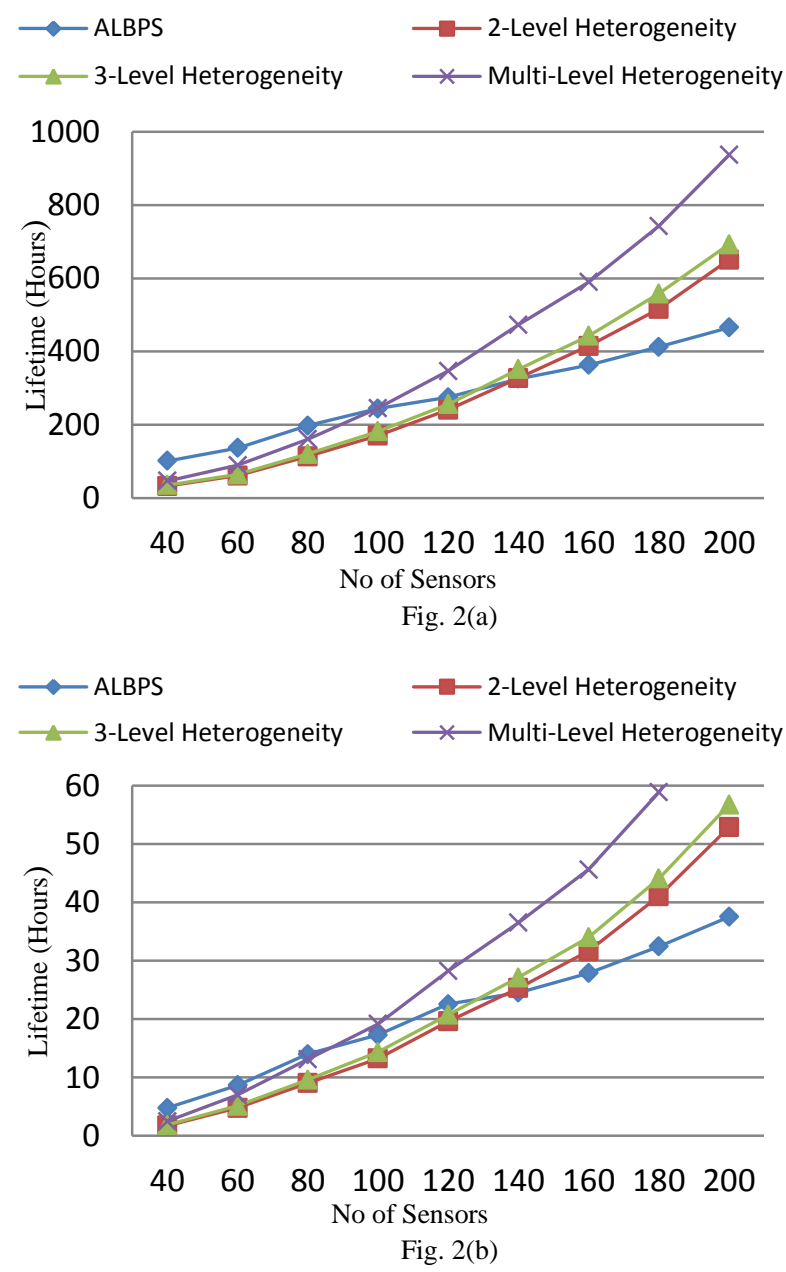

Fig. 2(a) \& (b): The total network Lifetime for different number of sensors at $60 \mathrm{M}$ adjustable sensing range and 50 Targets for Linear and Quadratic energy model $(\mathrm{m}=0.2$ and $\mathrm{a}=3$ for 2-level; $\mathrm{a}=1.5, \beta=3$, $\mathrm{m}=0.5$ and $\mathrm{m} 0=0.4$ for 3 -level)

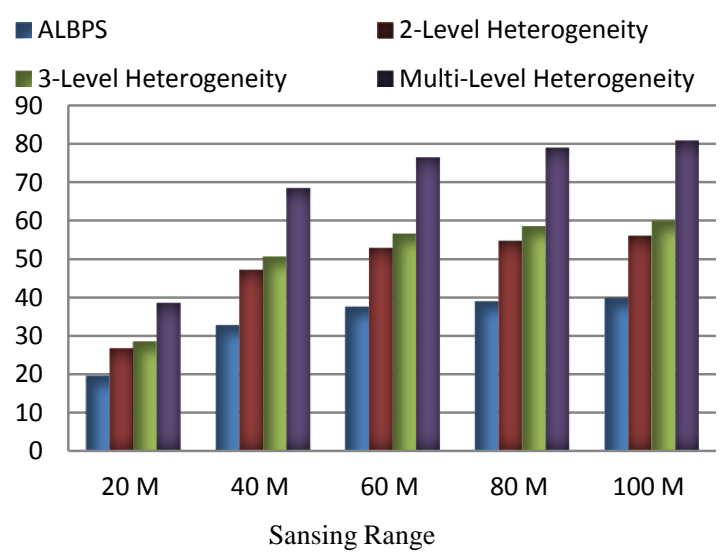

Fig. 3: Network lifetime for different adjustable sensing range values at 200 number of sensors, 50 Targets for quadratic energy model $(\mathrm{m}=0.2$ and $\mathrm{a}=3$ for 2 -level; $\mathrm{a}=1.5, \beta=3, \mathrm{~m}=0.5$ and $\mathrm{m} 0=0.4$ for 3 -level)
For case II, in 2-level heterogeneity, there are 20 advanced nodes with 5 times more energy than normal nodes. In 3-level heterogeneity, there are 20 super nodes deployed with 3 times more energy than normal nodes and 20 advance nodes deployed with 1 times more energy than the normal nodes. In multi-level heterogeneity, all the nodes have different level of energy within the interval of $[0.5,2]$.

The sensing range is associated with the energy level available with a sensor node. We observe from Figs. $3 \&$ 4 that as the sensing range increases the network lifetime also increases. Furthermore as the level of heterogeneity increases the lifetime also increases.

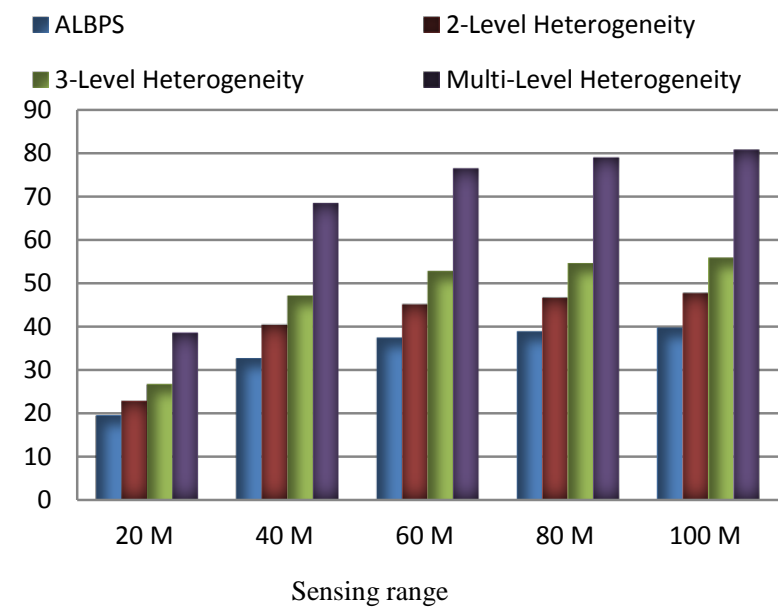

Fig. 4: Network lifetime for different adjustable sensing range values at 200 number of sensors, 50 Targets for quadratic energy model $(\mathrm{m}=0.1$ and $\mathrm{a}=5$ for 2 -level; $\mathrm{a}=1, \beta=3, \mathrm{~m}=0.2$ and $\mathrm{m} 0=0.5$ for 3 -level)

Now, we discuss the effect of maximum sensing range alone with number of targets on the lifetime of sensor network for 200 numbers of sensors which have been categorized above among different types of nodes based on the level of heterogeneity. We consider 25 and 50 targets randomly distributed and we vary sensing range $30 \mathrm{M}$ and $60 \mathrm{M}$ using linear and quadratic energy models.
ALBPS
—2-Level Heterogeneity
- 3-Level Heterogeneity
- Multi-Level Heterogeneity

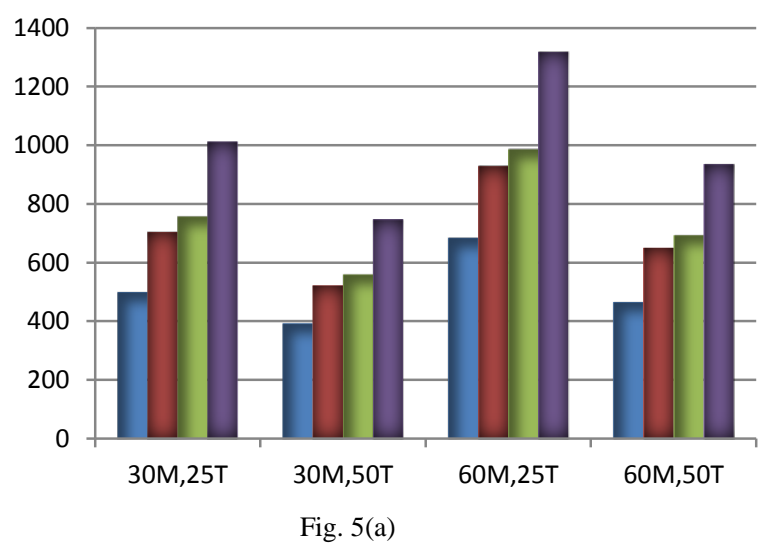




\section{ALBPS}

3-Level Heterogeneity

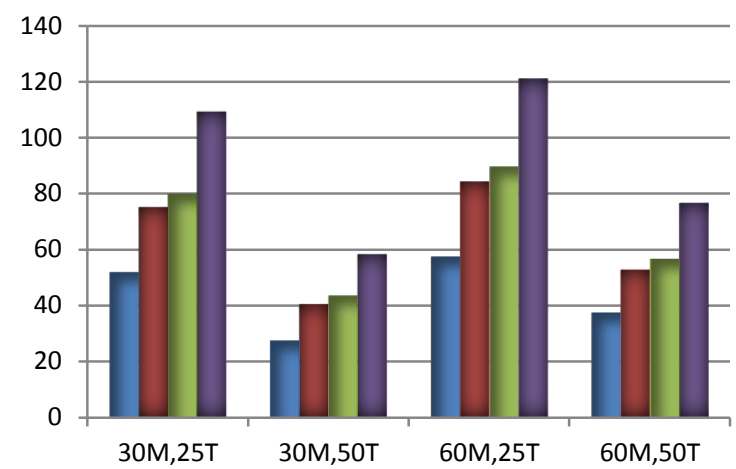

Fig. 5(b)

Fig. 5(a) \& (b): Network lifetime for different adjustable sensing range and target values at 200 number of sensors for linear and quadratic energy models $(\mathrm{m}=0.2$ and $\mathrm{a}=3$ for 2 -level; $\mathrm{a}=1.5, \beta=3, \mathrm{~m}=0.5$ and $\mathrm{m} 0=0.4$ for 3 -level)

\section{ALBPS \\ - 2-Level Heterogeneity \\ ם3-Level Heterogeneity \\ - Multi-Level Heterogeneity}

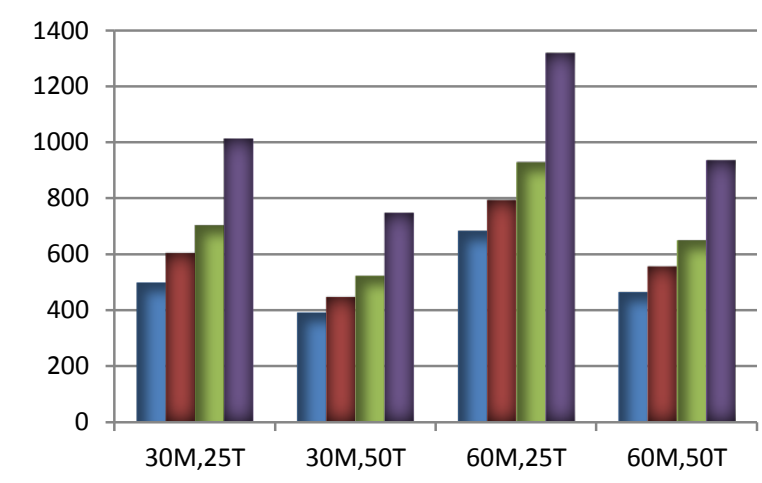

Fig. 6(a)

\section{ALBPS}

a-Level Heterogeneity

- 2-Level Heterogeneity

aulti-Level Heterogeneity

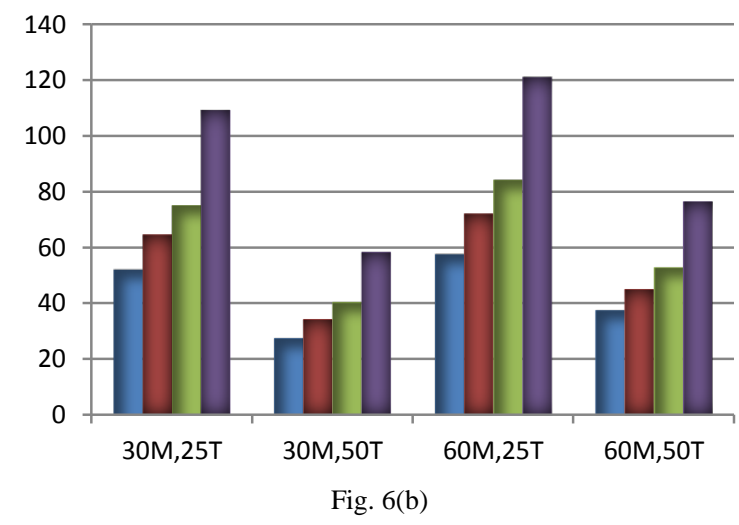

Fig. 6(a) \& (b): Network lifetime for different adjustable sensing range and target values at 200 number of sensors for Linear and Quadratic energy model $(\mathrm{m}=0.1$ and $\mathrm{a}=5$ for 2 -level; $\mathrm{a}=1, \beta=3, \mathrm{~m}=0.2$ and $\mathrm{m} 0=0.5$ for 3 -level)

We observe from Figs. 5(a) and (b) that increasing the number of targets, the lifetime of a sensor network decreases for the given sensing range. In this case also as the level of heterogeneity increases, the lifetime increases. We obtain similar results for case II as shown in Figs. 6(a) and (b). The results are consistent with the previous results because the network lifetime increases with the decrease in the number of targets.

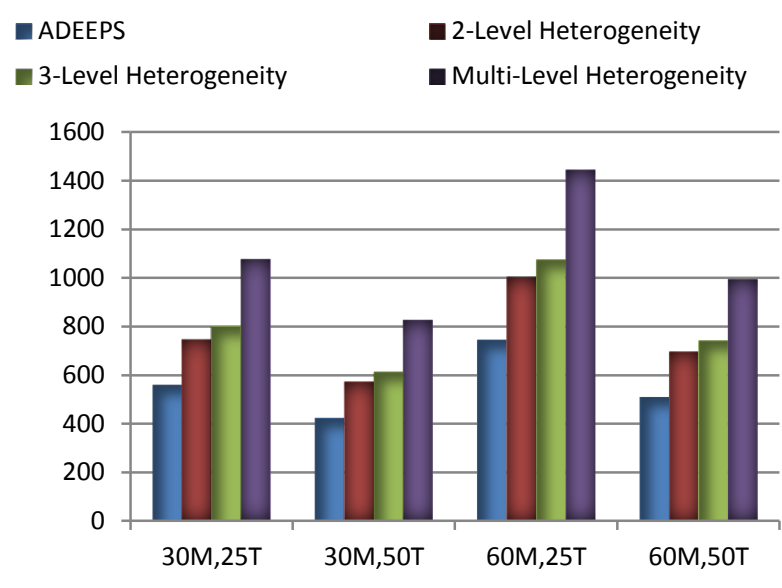

Fig. 7(a)

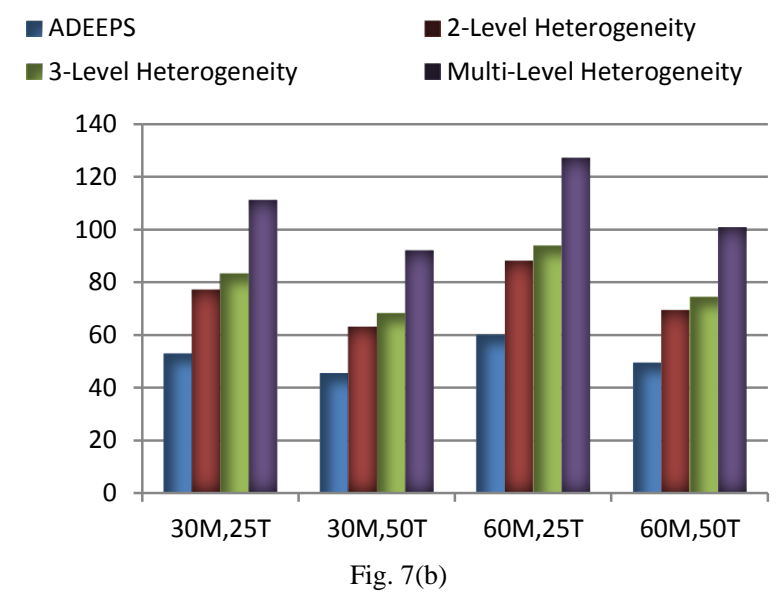

Fig. 7(a) \& (b): Network lifetime for different adjustable sensing range and target values at 200 number of sensors for Linear and Quadratic energy model $(\mathrm{m}=0.2$ and $\mathrm{a}=3$ for 2 -level; $\mathrm{a}=1.5, \beta=3, \mathrm{~m}=0.5$ and $\mathrm{m} 0=0.4$ for 3 -level)
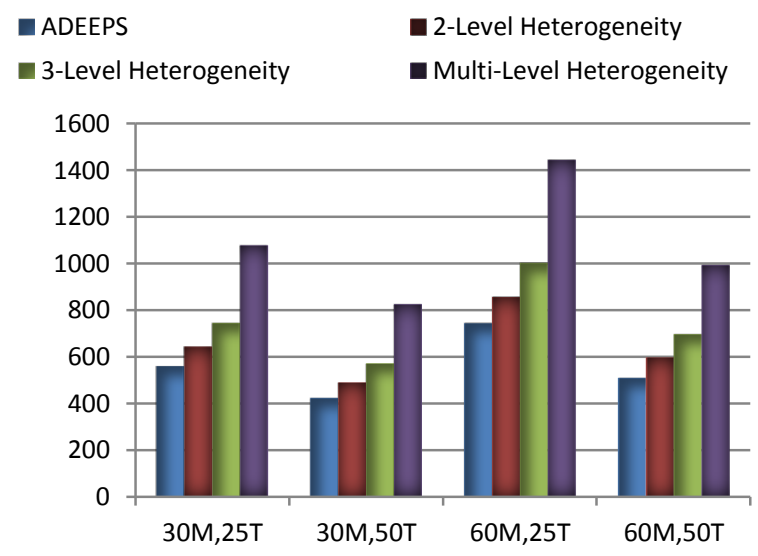

Fig. 8(a) 


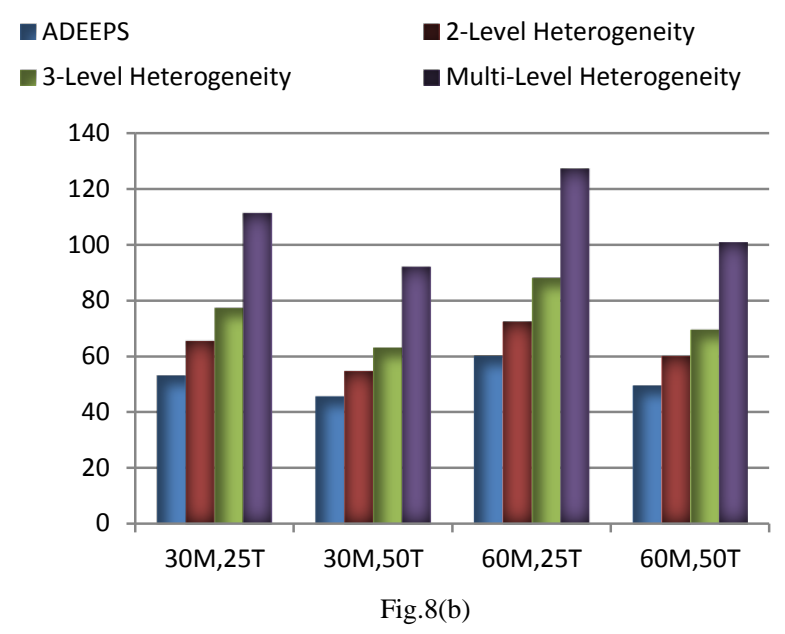

Fig. 8(a) \&( b): Network lifetime for different adjustable sensing range and target values at 200 number of sensors for Linear and Quadratic energy model $(\mathrm{m}=0.1$ and $\mathrm{a}=5$ for 2 -level; $\mathrm{a}=1, \beta=3, \mathrm{~m}=0.2$ and $\mathrm{m} 0=0.5$ for 3 -level)
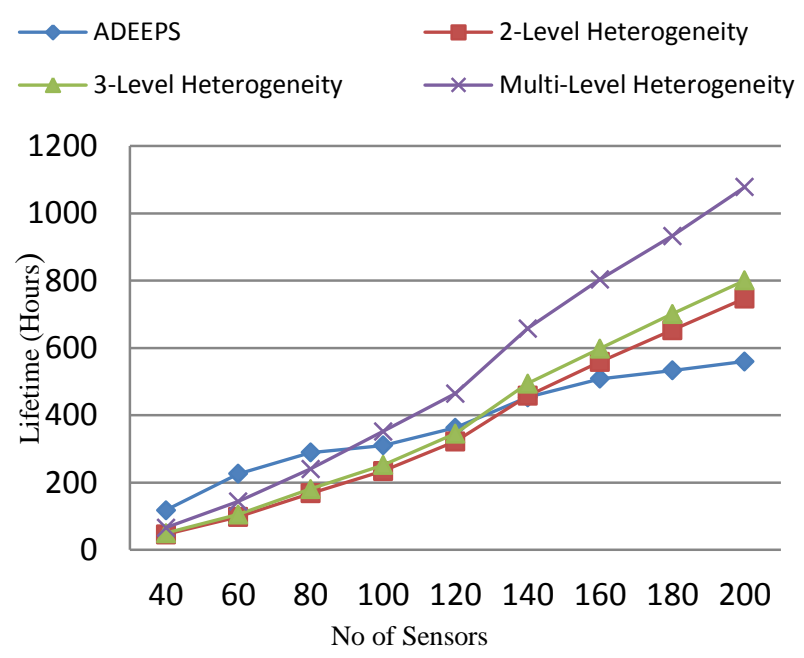

Fig. 9(a)
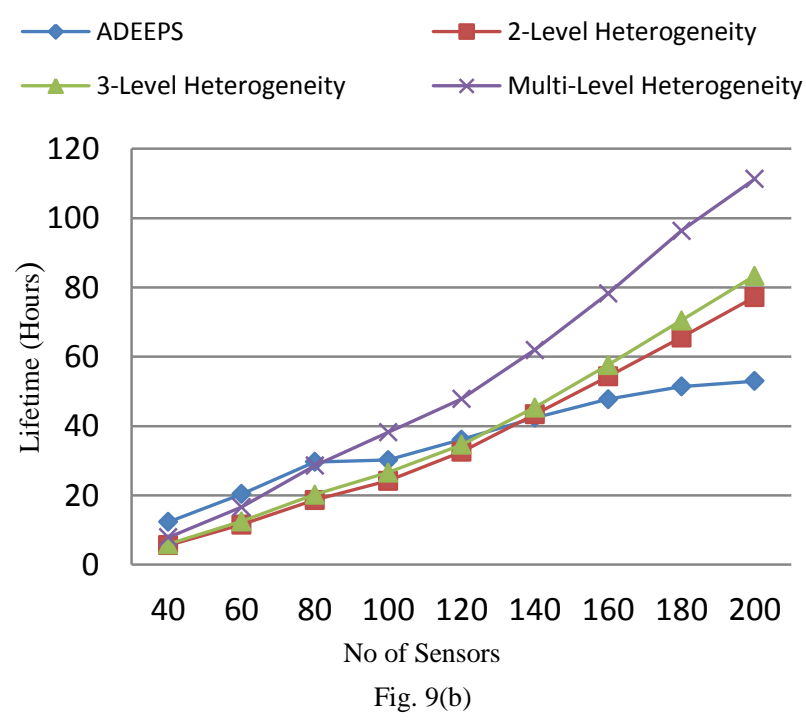

Fig. 9(a) \& (b): The total network Lifetime for different number of sensors at $30 \mathrm{M}$ adjustable sensing range and 25 Targets for Linear and

Quadratic energy model $(\mathrm{m}=0.2$ and $\mathrm{a}=3$ for 2 -level; $\mathrm{a}=1.5, \beta=3$, $\mathrm{m}=0.5$ and $\mathrm{m} 0=0.4$ for 3 -level)
For ADEEPS with heterogeneity, we have obtained similar results as obtained for ALBPS with heterogeneity. The corresponding results are shown in Figs. 7(a)-(b), 8(a)-(b), and 9(a)-(b). The protocols ALBPS and ADEEPS [4] are for homogeneous networks. Therefore, it is not justifiable to compare their performance with that of the proposed ones as they are meant for heterogeneous networks. However, for the sake of reference, their simulation results have also been given along with that of the proposed ones.

\section{CONCLUSION}

In this paper, we have discussed two new protocols by incorporating different levels of heterogeneity in ALPBS and ADEEPS. The outcome of the paper can be summarized as follows. The proposed protocols perform better than their homogeneous counterpart for all levels of heterogeneity. As the level of heterogeneity increases, the lifetime also increases. For the given number of targets and sensing ranges, the network lifetime increases with the number of sensors. When the number of targets is increased, the network lifetime decreases as more targets are monitored by the fixed number of sensors.

\section{REFERENCES}

[1] P. Berman, G. Calinescu, C. Shah and A. Zelikovsky, "Power Efficient Monitoring Management in Sensor Networks," IEEE Wireless Communication and Networking Conference (WCNC'04), pp. 2329-2334, Atlanta, March 2004.

[2] M. Cardei, M.T. Thai, Y. Li, and W. Wu, "Energyefficient target coverage in wireless sensor networks", In Proc. of IEEE Infocom, 2005.

[3] M. Cardei, J. Wu, M. Lu, "Improving network lifetime using sensors with adjustable sensing ranges", International Journal of Sensor Networks, (IJSNET), Vol. 1, No. 1/2, 2006.

[4] Brinza, D. and Zelikovsky, A, "DEEPS: Deterministic Energy-Efficient Protocol for Sensor networks", ACIS International Workshop on Self-Assembling Wireless Networks (SAWN'06), Proc. of SNPD, pp. 261-266, 2006.

[5] A. Dhawan, A. Aung and S. K. Prasad, "Distributed Scheduling of a Network of Adjustable Range Sensors for Coverage Problems", In ICISTM'2010, International Conference on Information Systems, Technology and Management, 2010 , Springer Link Book Chapter ,Vol. 54, Pages 123-132

[6] Dilip Kumar, T. S. Aseri, R. B. Patel "EEHC: Energy Efficient Heterogeneous Clustered Scheme for Wireless Sensor Networks", International Journal of Computer Communications, Elsevier, 32(4): 662-667, March 2009.

[7] Yingchi Mao, Zhen Liu, Lili Zhang, Xiaofang Li, "An Effective Data Gathering Scheme in Heterogeneous Energy Wireless Sensor Networks," CSE, vol. 1, pp.338343, International Conference on Computational Science and Engineering, 2009.

[8] Q. Li, Z. Qingxin, and W. Mingwen, "Design of a Distributed Energy Efficient Clustering Algorithm for Heterogeneous Wireless Sensor Networks", Computer Communications, vol. 29, pp. 2230-7, 2006.

[9] Satish Chand, Samayveer Singh and Bijendra Kumar, "Heterogeneous HEED Protocol for Wireless Sensor 
Networks", Wireless Personal Communications, vol. 77, issue 3, pp. 2117-2139, Feb 2014.

[10] Samayveer Singh, Satish Chand and Bijendra Kumar, "3Tier Heterogeneous Network model for Increasing Lifetime in Three Dimensional WSNs," Quality, Reliability, Security and Robustness in Heterogeneous Networks, Lecture Notes of the Institute for Computer Sciences, Social Informatics and Telecommunications Engineering, vol. 115, pp 238-247, 2013.

[11] D. Kumar, T. C. Aseri and R. B. Patel, "A Novel Multihop Energy Efficient Heterogeneous Clustered Scheme for Wireless Sensor Networks," Tamkang Journal of Science and Engineering, vol. 14, no. 4, pp. 359-368, 2011.

[12] Samayveer Singh, Satish Chand and Bijendra Kumar, "3Level Heterogeneity Model for Wireless Sensor Networks," Int. Journal of Computer Network and Information Security (IJCNIS), vol. 5, no. 4, pp.40-47, April 2013.

[13] Samayveer Singh and Ajay K Sharma, "Distributed Algorithms for Maximizing Lifetime of WSN with Heterogeneity and Adjustable Range for Different Deployment Strategies" I.J. Information Technology and Computer Science, vol.5, no.08, pp.101-108, 2013.

[14] Dilip Kumar, Trilok C. Aseri and R. B. Patel, "EECDA: Energy-Efficient Clustering and Data Aggregation Protocol for HeterogeneousWireless Sensor Networks," International Journal of Computers, Communication and Control, Romania, vol. 6, no.1, pp. 113-124, 2011.

[15] Satish Chand, Samayveer Singh, and Bijendra Kumar, "hetADEEPS: ADEEPS for Heterogeneous Wireless Sensor Networks" International Journal of Future Generation Communication and Networking, vol.6, no.5, pp.21-32, 2013.

\section{Authors' Profiles}

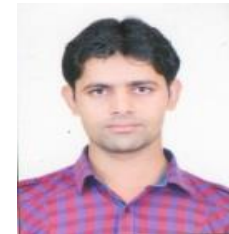

Samayveer Singh received his B.Tech. in Information Technology from Uttar Pradesh Technical University, Lucknow, India in 2007, and his M.Tech. degree in Computer Science \& Engineering from National Institute of Technology, Jalandhar, India, in 2010. He is now a PhD student of Department of Computer Engineering at Netaji Subhas Institute of Technology, New Delhi, India. His research interest includes wireless sensor networks.

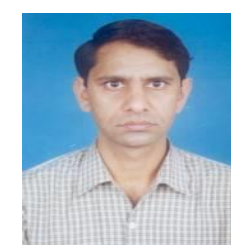

Satish Chand did his M.Sc. in Mathematics from Indian Institute of Technology, Kanpur, India and M.Tech. in Computer Science from Indian Institute of Technology, Kharagpur, India and Ph.D. from Jawaharlal Nehru University, New Delhi, India. Presently he is Professor in Computer Engineering Division at Netaji Subhas Institute of Technology, Delhi, India. Areas of his research interest are Multimedia Broadcasting, Networking, Video-on-Demand, Cryptography, and Image processing.

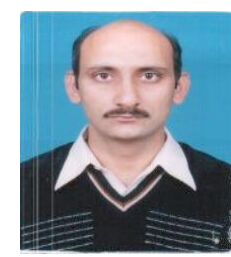

Bijendra Kumar did his Bachelor of Engineering from H.B.T.I. Kanpur, India. He has done his Ph.D. in Delhi University, Delhi, India in 2011. Presently he is Assistant Professor in Computer Engineering Division at Netaji Subhas Institute of Technology, Delhi, India. His areas of research interests are Video applications, watermarking, and Design of algorithms.

How to cite this paper: Samayveer Singh, Satish Chand, Bijendra Kumar,"Performance Evaluation of Distributed Protocols Using Different Levels of Heterogeneity Models in Wireless Sensor Networks", IJCNIS, vol.7, no.1, pp.38-45, 2015. DOI: $10.5815 /$ ijcnis.2015.01.06 